\title{
Disruption index depends on length of citation window
}

\author{
Lutz Bornmann; Alexander Tekles
}

How to cite this article:

Bornmann, Lutz; Tekles, Alexander (2019). "Disruption index depends on length of citation window". El profesional de la información, v. 28, n. 2, e280207.

https://doi.org//10.3145/epi.2019.mar.07

Article received on 18 March 2019

Approved on 25 March 2019

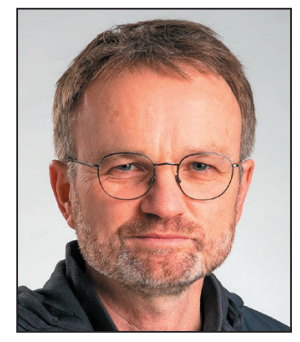

Lutz Bornmann $\triangle$

https://orcid.org/0000-0003-0810-7091

Administrative Headquarters of the Max

Planck Society, Division for Science and

Innovation Studies

Hofgartenstr., 8. 80539 Munich, Germany

bornmann@gv.mpg.de

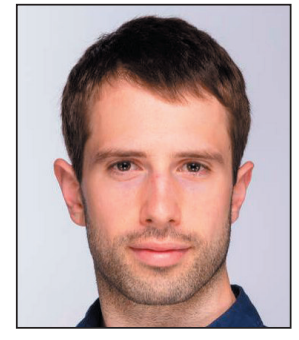

Alexander Tekles

https://orcid.org/0000-0001-8765-9331

Administrative Headquarters of the Max Planck Society, Division for Science and Innovation Studies

Hofgartenstr., 8. 80539 Munich, Germany alexander.tekles.extern@gv.mpg.de and

Ludwig-Maximilians-University Munich, Department of Sociology Konradstr. 680801 Munich, Germany

\begin{abstract}
In the context of recent developments in scientometrics to measure novelty or creative potential, Wu, Wang, and Evans (2019) propose a new disruption index that measures the extent to which a publication disrupts the field of science. We calculated the disruption index for some example papers. The analyses of the index values (using our Web of Science in-house database) show that they depend on the citation window (the period of time over which citations are collected).
\end{abstract}

\section{Keywords}

Bibliometrics; Bibliometric indicators; Citation window; Disruption index; Novelty; Measuring methods; Scientific impact.

\section{Introduction}

Citation counts measure the usefulness of research but cannot point towards exceptional research that revolutionizes our way of thinking. Seven of the 10 most cited publications of all time relate to biological lab techniques (Van-Noorden; Maher; Nuzzo, 2014).

In the context of recent developments in scientometrics to measure novelty or creative potential (Lee; Walsh; Wang, 2015; Uzzi; Mukherjee; Stringer; Jones, 2013), Wu et al. (2019) propose a new disruption index that measures the extent to which a publication disrupts the field of science. The index varies between values of -1 and 1 , corresponding to work that develops (by broadcasting the importance of prior research) or disrupts (weakening prior research by receiving all later attention), respectively.

\section{Citation windows of at least 3 years are needed}

We calculated the disruption index for the example papers used for illustration purposed by Wu et al. (2019) in their Figure 1. The analyses of the index values (using our Web of Science in-house database) show that they depend on the citation window (the period of time over which citations are collected). This dependence is shown for two example papers (Davis et al., 1995; Randall; Sundrum, 1999) from Wu et al. (2019) in Figure 1.
Wu et al. (2019) propose a new disruption index that measures the extent to which a publication disrupts the field of science 
Whereas the disruption index is more or less stable over time for Randall and Sundrum (1999), Davis et al. (1995) only achieved a stable value five years after publication.

We calculated the disruption index for two other examples - the most cited papers in Nature from the 1990s (Iijima, 1991; O'Regan; Grätzel, 1991). It is interesting to observe that the disruptive potential of both papers only appears several years after their publication. In the first few years, both papers seem to be balanced between disruption and development.

In bibliometrics, it is standard practice to use a citation window of at least three years (Bornmann, in press). Our results for the disruption index reveal that it would also appear to be necessary to

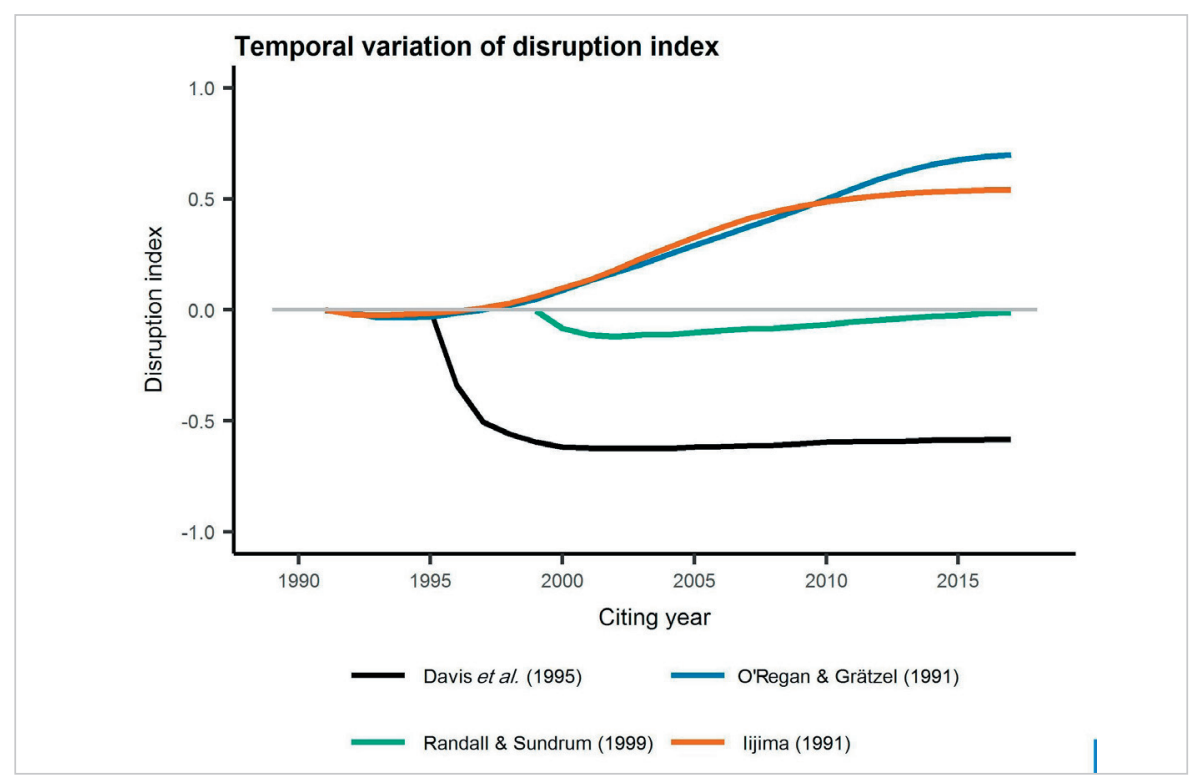

Figure 1. Dependence of the disruption index on the citation window have recommendations for an appropriate citation window. We assume that a citation window of at least three years is necessary to produce meaningful results. However, as our analyses show, this may not suffice in some cases. Further research into the properties of the promising disruption index proposed by $\mathbf{W u}$ et al. (2019) is thus important for its appropriate use in bibliometrics.

Disruption index depends on the citation window (the period of time over which citations are collected)

\section{References}

Bornmann, Lutz (in press). "Bibliometric indicators - methods for measuring science". In: R. Williams (ed.). Encyclopedia of research methods. Thousand Oaks, CA, USA: Sage.

Davis, Kendall B.; Mewes, Marc-Oliver; Andrews, Michael R.; Van-Druten, Nicolaas J.; Durfee, Dallin S.; Stamper-Kurn, Dan M.; Ketterle, Wolfgang (1995). „Bose-Einstein condensation in a gas of sodium atoms. Physical review letters, v. 75, n. 22, pp. 3969-3973.

https://doi.org/10.1103/PhysRevLett.75.3969

lijima, Sumio (1991). “Helical microtubules of graphitic carbon”. Nature, v. 354, pp. 56-58.

https://doi.org/10.1038/354056a0

Lee, You-Na; Walsh, John P.; Wang, Jian (2015). "Creativity in scientific teams: Unpacking novelty and impact". Research policy, v. 44, n. 3, pp. 684-697.

https://doi.org/10.1016/j.respol.2014.10.007

O’Regan, Brian; Grätzel, Michael (1991). “A low-cost, high-efficiency solar cell based on dye-sensitized colloidal TiO films". Nature, v. 353, pp. 737-740.

https://doi.org/10.1038/353737a0

Randall, Lisa; Sundrum, Raman (1999). "Large mass hierarchy from a small extra dimension”. Physical review letters, v. 83, n. 17, pp. 3370-3373.

https://doi.org/10.1103/PhysRevLett.83.3370

Uzzi, Brian; Mukherjee, Satyam; Stringer, Michael; Jones, Ben (2013). "Atypical combinations and scientific impact". Science, v. 342, n. 6157, pp. 468-472.

https://doi.org/10.1126/science.1240474

Van-Noorden, Richard; Maher, Brendan; Nuzzo, Regina L. (2014). “The Top 100 papers”. Nature, v. 514, pp. 550-553. https://www.nature.com/news/the-top-100-papers-1.16224

Wu, Lingfei; Wang, Dashun; Evans, James A. (2019). "Large teams develop and small teams disrupt science and technology". Nature, v. 566, pp. 378-382.

https://doi.org/10.1038/s41586-019-0941-9 